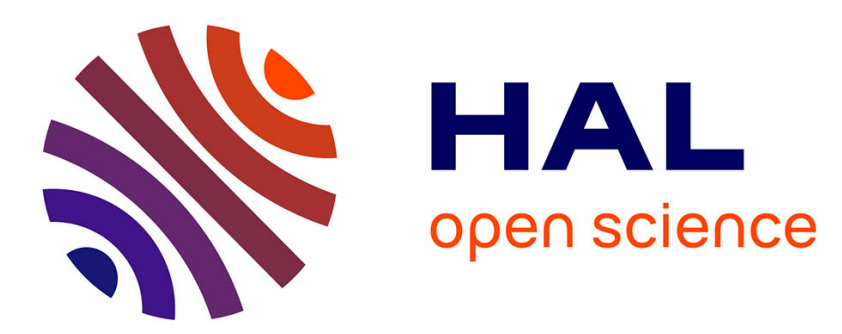

\title{
Geophysical Observations of Phobos Transits by InSight
}

S. Stähler, R. Widmer-schnidrig, J.-r. Scholz, M. Driel, A. Mittelholz, K. Hurst, C. Johnson, M. Lemmon, P. Lognonné, R. Lorenz, et al.

\section{To cite this version:}

S. Stähler, R. Widmer-schnidrig, J.-r. Scholz, M. Driel, A. Mittelholz, et al.. Geophysical Observations of Phobos Transits by InSight. Geophysical Research Letters, 2020, 47 (19), 10.1029/2020GL089099 . hal-03583301

\section{HAL Id: hal-03583301 \\ https://hal.science/hal-03583301}

Submitted on 24 Feb 2022

HAL is a multi-disciplinary open access archive for the deposit and dissemination of scientific research documents, whether they are published or not. The documents may come from teaching and research institutions in France or abroad, or from public or private research centers.
L'archive ouverte pluridisciplinaire HAL, est destinée au dépôt et à la diffusion de documents scientifiques de niveau recherche, publiés ou non, émanant des établissements d'enseignement et de recherche français ou étrangers, des laboratoires publics ou privés. 


\section{Geophysical Research Letters}

\author{
RESEARCH LETTER \\ 10.1029/2020GL089099 \\ Special Section: \\ InSight at Mars
}

Key Points:

- Multiple geophysical InSight instruments observe unexpected signals during Phobos transits

- Local ground deformation due to surface temperature drops explain the tilt signals seen by the seismometer

- The drop in the solar array currents results in a change of the magnetic field

Supporting Information:

- Supporting Information S1

Correspondence to:

S. C. Stähler,

simon.staehler@erdw.ethz.ch

\section{Citation:}

Stähler, S. C., Widmer-Schnidrig, R., Scholz, J.-R., van Driel, M., Mittelholz, A., Hurst, K., et al. (2020). Geophysical observations of Phobos transits by InSight. Geophysical Research Letters, 47, e2020GL089099. https://doi.org/ 10.1029/2020GL089099

Received 29 MAY 2020

Accepted 27 JUL 2020

Accepted article online 4 AUG 2020

(C)2020. American Geophysical Union. All Rights Reserved.

\section{Geophysical Observations of Phobos Transits by InSight}

\author{
S. C. Stähler ${ }^{1}$ iD, R. Widmer-Schnidrig ${ }^{2}$ iD, J.-R. Scholz ${ }^{3}$ iD, M. van Driel ${ }^{1}$ iD, A. Mittelholz ${ }^{4}$ iD, \\ K. Hurst ${ }^{5}$ iD, C. L. Johnson ${ }^{4,6}$ D, M. T. Lemmon ${ }^{7}$ iD, P. Lognonné ${ }^{8}$ iD, R. D. Lorenz 9 iD, \\ N. T. Müller ${ }^{10}$ iD, L. Pou ${ }^{11}$ iD, A. Spiga ${ }^{12,13}$ iD, D. Banfield ${ }^{14}$ (D) S. Ceylan ${ }^{1}$ (D, C. Charalambous ${ }^{15}$ (D), \\ J. Clinton ${ }^{16}$ (D) D. Giardini ${ }^{1}$ iD, F. Nimmo ${ }^{11}$ iD, M. Panning ${ }^{5}$ (D) W. Zürn ${ }^{17}$ (D) and \\ W. B. Banerdt ${ }^{5}$ iD
}

${ }^{1}$ Institute of Geophysics, ETH Zürich, Zürich, Switzerland, ${ }^{2}$ Black Forest Observatory, Institute of Geodesy, Stuttgart University, Wolfach, Germany, ${ }^{3}$ Max Planck Institute for Solar System Research, Göttingen, Germany, ${ }^{4}$ Department of Earth, Ocean and Atmospheric Sciences, University of British Columbia, Vancouver, British Columbia, Canada, ${ }^{5}$ Jet Propulsion Laboratory, California Institute of Technology, Pasadena, CA, USA, ${ }^{6}$ Planetary Science Institute, Tucson, AZ, USA, ${ }^{7}$ Space Science Institute, Boulder, CO, USA, ${ }^{8}$ Université de Paris, Institut de physique du globe de Paris, CNRS, Paris, France, ${ }^{9}$ Johns Hopkins Applied Physics Laboratory, Laurel, MD, USA, ${ }^{10}$ German Aerospace Center (DLR), Institute of Planetary Research, Berlin, Germany, ${ }^{11}$ Department of Earth and Planetary Sciences, University of California Santa Cruz, Santa Cruz, CA, USA, ${ }^{12}$ Laboratoire de Météorologie Dynamique / Institut Pierre Simon Laplace (LMD/IPSL), Sorbonne Université, Centre National de la Recherche Scientifique (CNRS), École Polytechnique, École Normale Supérieure (ENS), Campus Pierre et Marie Curie BC99, Paris, France, ${ }^{13}$ Institut Universitaire de France, Paris, France, ${ }^{14}$ Cornell Center for Astrophysics and Planetary Science, Cornell University, Ithaca, NY, USA, ${ }^{15}$ Department of Electrical and Electronic Engineering, Imperial College London, London, UK, ${ }^{16}$ Swiss Seismological Service (SED), ETH Zürich, Zürich, Switzerland, ${ }^{17}$ Black Forest Observatory, KIT Karlsruhe, Wolfach, Germany

\begin{abstract}
Since landing on Mars, the NASA InSight lander has witnessed eight Phobos and one Deimos transits. All transits could be observed by a drop in the solar array current and the surface temperature, but more surprisingly, for several ones, a clear signature was recorded with the seismic sensors and the magnetometer. We present a preliminary interpretation of the seismometer data as temperature-induced local deformation of the ground, supported by terrestrial analog experiments and finite-element modeling. The magnetic signature is most likely induced by changing currents from the solar arrays. While the observations are not fully understood yet, the recording of transit-related phenomena with high sampling rate will allow more precise measurements of the transit times, thus providing additional constraints for the orbital parameters of Phobos. The response of the seismometer can potentially also be used to constrain the thermoelastic properties of the shallow regolith at the landing site.
\end{abstract}

Plain Language Summary The geophysical lander, InSight, has been operating on the surface of Mars since November 2018. Since then, the Martian moons Phobos and Deimos have been partially blocking the Sun, as seen from the InSight landing site, multiple times. Multiple InSight instruments have been measuring the effect of those transits; this surprisingly includes the seismometer and the magnetometer. We conclude that temperature-induced deformation and tilt are responsible for the seismic measurements. The change observed in the magnetometer measurements are most likely the result of a drop in the solar array currents. We do not observe atmospheric modulations with InSight's weather station during the transit. These observations help constrain orbital parameters of the Martian moons, and the seismometer signal might allow investigating thermoelastic properties of the shallow Martian material.

\section{Introduction}

The small Martian satellites Phobos and Deimos orbit in synchronous rotation with inclinations of less than $1^{\circ}$ (Grier \& Rivkin, 2019). For observers, or robot landers at near-equatorial latitudes, it is therefore possible to observe solar transits of both satellites (Ledger, 1879), in blocks of up to five transits twice per Martian year. The first observation of a transit from the surface of Mars was done by using the scan camera on the Viking 1 lander as a brightness detector (Christou, 2002; Duxbury, 1978). The first actual image of Phobos and Deimos transiting the Sun was captured by the Spirit and Opportunity rovers (Mars Exploration Rovers A and B) in 2004 (Bell et al., 2005). The determination of transit timing allows updating ephemeris tables, which are crucial for missions targeting the moons (Usui et al., 2018). They also determine the 
moons' secular acceleration, from which the tidal dissipation in the Martian mantle can be estimated (Bills et al., 2005; Khan et al., 2018; Nimmo \& Faul, 2013). Further observations of Phobos transits from fixed reference positions on the surface are therefore highly desired.

Here we discuss the main Phobos and Deimos transits experienced by the InSight lander in the first 500 sols since its arrival at the surface of Mars on 26 November 2018 (Banerdt et al., 2020). InSight features a fully deployed shielded seismometer on the surface of Mars (Lognonné et al., 2019, 2020), as well as sensors probing the atmospheric, magnetic, and surface environments (Banfield et al., 2018, 2020). Furthermore, of interest for transit studies, the InSight lander has been located independently from orbital imaging and the radio tracking experiment RISE onboard (Folkner et al., 2018) and can be considered the best-constrained location on the planet at $4.50238417^{\circ} \mathrm{N}, 135.62344690^{\circ} \mathrm{E}$, at an elevation of $-2,613.426 \mathrm{~m}$ with respect to the MOLA areoid (ground under spacecraft deck center; see Golombek et al., 2020).

Rapid change in irradiation by moving cloud shadows is known to correlate with tilt-like signals on surface-installed seismometers. While no literature on the topic seems to exist, it has been described by operators of temporary networks despite extensive local thermal shielding (Karin Sigloch, personal communication, 2012). Schweitzer et al. (2014) mitigated horizontal low-frequency noise at the Antarctic station TROLL by covering the granite surface surrounding the seismometer with loose rocks to shield the bed rock from direct sunlight, using insights from experiments with transient heat sources on a well shielded vault seismometer (Zürn \& Otto, 2000). While not changing the thermal insulation of the seismometer, these measures had a large impact on low-frequency seismic noise levels.

Earth observations have shown that solar eclipses can lead to changes in ionospheric currents, due to a drop in electron density of 35\% (Huba \& Drob, 2017; Reinisch et al., 2018). On Earth, the effect on the geomagnetic field varies depending on the solar conditions and activity level in the external fields and is often too subtle to be detected on an individual eclipse, requiring statistical analyses of many events (Kim \& Chang, 2018).

We first discuss the observations of the individual instruments and present a common explanation framework afterward.

\section{Observations}

\subsection{Observations With Solar Arrays}

The most immediate surface manifestation of an eclipse or a transit on Earth or Mars is the drop in sunlight due to the geometric obstruction of part of the solar disk. For Phobos, this can reach around $40 \%$. The current generated by the InSight solar arrays is monitored when the lander is awake and is recorded with a precision of about $0.1 \%$ (for noon sunlight levels) at a sample interval of around $30 \mathrm{~s}$ until September 2019 and $4 \mathrm{~s}$ afterward (Lorenz, Lemmon, Maki, et al., 2020).

A 120-s transit of Deimos with a transit depth (change in brightness) of 1\% was observed on Sol 481 (Lorenz, Lemmon, \& Mueller, 2020). Phobos transit depths are usually deeper but briefer, and the drops in solar array currents are lower than the predicted geometric obstructions. Observed depths on Sols 495, 498, 499, and 501 were $2 \%, 10 \%, 10 \%$, and $19 \%$ respectively (see Table 1). The difference is assumed to reflect the contribution of light scattered by dust in the sky outside of the Phobos shadow, which makes a more significant relative contribution when the Sun is low. Since scattered light affects the surface heat budget in the same way as direct sunlight, the solar array current is then a useful measure of the total solar forcing for modeling any thermal effects.

The Sol 96-99 Phobos transits of spring 2019 were detected as single-sample current drops, confirming their occurrence but precluding quantitative analysis (Lorenz, Lemmon, Maki, et al., 2020).

\subsection{Observations With the Seismometer}

Three of the six Phobos transits that occurred between InSight landing and today (not counting grazing transits) produced some observable signal on the two sensors of SEIS; the very broadband seismometer (VBB) and the short-period seismometer (SP), which share one isolating housing (RWEB) below a wind and thermal shield (WTS) (Lognonné et al., 2019). Table 1 lists all transits (green, clear signal above noise level; orange, increased noise level but signal still observable; red, no clear signal). After correction for 


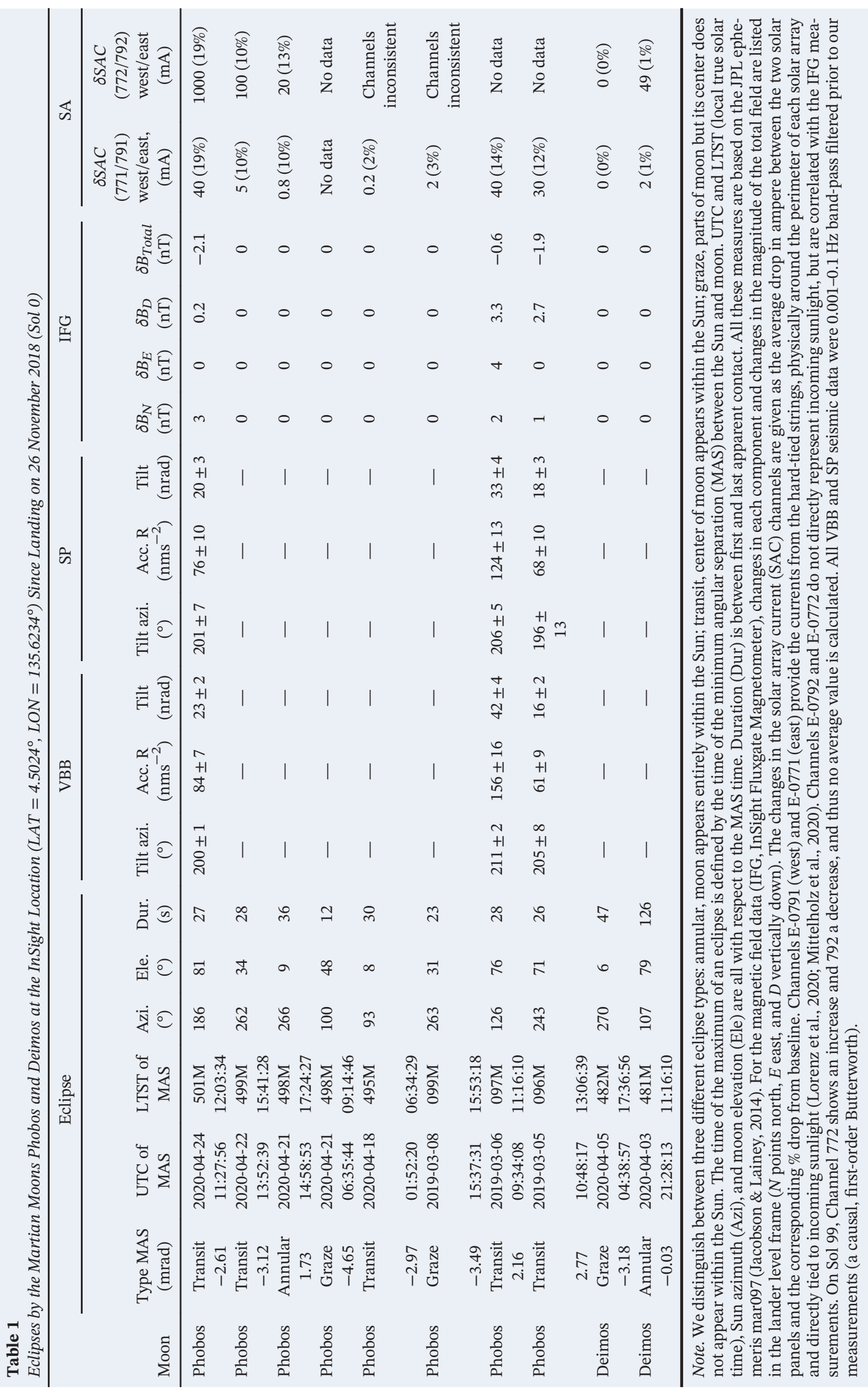


instrument response, these three transits all have a clear signal with an acceleration amplitude of $\sim 50$ $\mathrm{nms}^{-2}$ and a duration of $\sim 100 \mathrm{~s}$. Rotation into a ZNE coordinate system shows a first pulse of positive polarity on both horizontal components with a duration of $30 \mathrm{~s}$, followed by a decaying part of about $70 \mathrm{~s}$. There is no clear signal on the vertical component for any transit event.

All three transits seen by VBB and SP occur around midday local true solar time (LTST) when the Sun is high in the sky, while all other eclipses not observed by VBB and SP do not occur around noon LTST. For the ones with discernible seismic signal, the corresponding tilt azimuths (determined via linear polarization analysis, e.g., Scholz et al., 2017) point away from the connector of the tether connecting SEIS to the lander, which is located at an azimuth $\sim 15^{\circ}$. Tilt angles $\theta$ were derived from the peak-to-peak amplitude of filtered radial acceleration data during the transit $\left(a_{R}\right)$, following $\sin (\theta) \approx \theta=a_{R} / g_{\text {Mars }}$, with $g_{\text {Mars }}=$ $3.71 \mathrm{~ms}^{-2}$. Acceleration and tilt errors, estimated via the data standard deviation using a 300-s window before and after the transit, are about $10 \%$. No Deimos transit produces any clear signal on SEIS (Table 1). A measurement example for the Phobos transit on Sol 501 is given in Figure S2 in the Supporting Information.

The onset of the VBB signal is delayed by $5 \mathrm{~s}$, relative to the first contact of Phobos with the Sun and also with respect to the solar array current and the magnetometer channels (see below).

\subsection{Observations With the Magnetometer}

The magnetic field at the InSight landing site has a static contribution with an amplitude of $2013 \mathrm{nT}$ due to the crustal magnetic field and a time-varying contribution of $\sim 10-100 \mathrm{nT}$ due to natural (e.g., ionosphere) and artificial sources (Johnson et al., 2020; Mittelholz et al., 2020). We assess the magnetic field signals associated with all listed transits (Table 1). For the earlier transits (up to Sol 99), the InSight Fluxgate Magnetometer (IFG) data are sampled at $0.2 \mathrm{~Hz}$; for the later transits, the data rates are increased to $2 \mathrm{~Hz}$. Magnetic field perturbations are seen associated with Phobos transits on Sols 96, 97, and 501 (Figure S1), with different relative contributions from each component for individual transits but an overall decrease in the magnetic field amplitude. All other transits did not result in a detectable magnetic field signal (Table 1).

We investigated possible mechanisms that could cause the observed response, focusing on three aspects: (1) the IFG response to temperature and solar array current fluctuations, (2) tilt as a result of differential contraction of the lander legs, and (3) an ionospheric response.

\subsubsection{Temperature and Solar Array Current}

The IFG is affected by changes in temperature and solar array currents. The data processing pipeline attempts to correct for these effects, particularly on diurnal time scales. However, the lack of a prelaunch magnetic cleanliness program or comprehensive calibrations means that small residual effects may still be present (Joy et al., 2019; Mittelholz et al., 2020). The IFG electronics and sensor temperatures showed no signals related to the transits, and we excluded those as possible reasons for the observed IFG response. However, the decrease in incoming sunlight resulted in changes in the solar array currents (Table 1). Transits that resulted in measurable magnetic fields responses are all associated with current drops larger than 0.01 A on Channels E-0771 and E-0791. The effect of solar array currents on the IFG data was not tested preflight; however, an order of magnitude calculation assuming a line current approximately $1 \mathrm{~m}$ from the IFG instrument indicates that a 3-nT change in the IFG data would require an $~ 0.015$-A drop in the current, which is approximately consistent with our observations.

2.3.2. Tilt

Differential thermal contractions of the three lander legs could lead to tilt of the lander deck and the IFG. At local noon, two legs are in full sunlight, while the third leg on the north side of the lander is partially shaded by the deck; this could possibly lead to the deck tilting south during the Phobos transit. However, a tilt of the magnetometer would not affect the magnitude of the overall signal but only of individual components. The IFG data during transits with a detectable signal do not support the tilt hypothesis as the magnitude of the magnetic field $|B|$ drops by up to $2.1 \mathrm{nT}$ during the transit (Table 1 ).

\subsubsection{Ionosphere}

The magnetic field can result from electric currents in the ionosphere, driven by atmospheric winds between $\sim 130$ and $\sim 180 \mathrm{~km}$ altitude. A drop in electron density associated with an eclipse might lead to changes in ionospheric currents. Drops up to $35 \%$ in electron density have been observed for solar 
eclipses on Earth (Huba \& Drob, 2017; Reinisch et al., 2018), but such eclipses last much longer ( $3 \mathrm{hr})$ than the transits discussed here. Thus, ionospheric effects are expected to be correspondingly smaller on Mars.

Although the magnetic field associated with the drops could be affected by changes in ionospheric currents, the signals that were recorded would require large, instantaneous responses, temporally correlated only with the Phobos shadow passing over the InSight landing site, which is implausible. Also, during a near-miss transit, no signal could be detected.

\subsection{Observations With InSight's Weather Station}

The InSight lander is equipped with a weather station capable of assessing atmospheric conditions for seismic observations (Banfield et al., 2018; Spiga et al., 2018). We assess the pressure, temperature, and wind signal associated with the three Phobos transits for which we observe both seismic and magnetic signals. For the earlier transits (up to Sol 99), pressure/wind-temperature measurements are available, sampled at $0.1 / 2 \mathrm{~Hz}$; for the later transits, the data rates are increased to $1 / 10 \mathrm{~Hz}$.

No particular distinctive signatures associated with the transits can be found in the atmospheric observations-even in the case of the major Phobos transit on Sol 501. The fluctuations of pressure, wind, and temperature during the transit are governed by the characteristic convective turbulence on Mars in the daytime hours (Banfield et al., 2020).

In the daytime hours prone to strong turbulence on Mars, two distinctive atmosphere-induced seismic signatures are found: broadband noise from wind and transient signatures associated with dust-devil-like convective vortices (Garcia et al., 2020; Kenda et al., 2020; Lognonné et al., 2020). We performed a vortex search following the approach detailed in Banfield et al. (2020) and Spiga et al. (2020). No convective vortex was detected during either the major Sol 501 Phobos transit or the Sols 96-97 transits, ruling out this seismic source. The Sol 501 Phobos transit actually occurred during a season at which the vortex encounters at the InSight landing site are significantly declined. Furthermore, wind noise due to turbulence during the three transits is uneventful, behaving like turbulent noise in normal conditions.

\subsection{Radiometer Observations}

The Heatflow and Physical Properties Package $\left(\mathrm{HP}^{3}\right)$ includes an infrared radiometer to monitor surface temperature in two spots approximately 1.5 and $3 \mathrm{~m}$ to the NNW of the lander deck center (Spohn et al., 2018). For an interval of $20 \mathrm{~min}$ around the transits on Sols 96, 97, 99, 497, 498, and 501, the radiometer observed with its maximum sampling rate of $0.46 \mathrm{~Hz}$. The $1 \sigma$ instrumental noise of the instrument is equivalent to a temperature difference of less than $0.25 \mathrm{~K}$ during the time of the transits (Mueller et al., 2020), and the temperature response to the transits is clearly visible (Figure 1). The temperature response is larger than expected based on preliminary calculations using the surface thermal inertia of $190 \pm 30 \mathrm{Jm}^{-2} \mathrm{~K}^{-1} \mathrm{~s}^{-1 / 2}$ derived from the the diurnal temperature curve (Golombek et al., 2020). This is similar to the remote observation of the temperature response to the transit by the Soviet Phobos ' 88 spacecraft, which was also larger than expected based on the diurnal temperature response (Betts et al., 1995). This is consistent with less dense and/or lower thermal conductivity material in the upper millimeter compared to the centimeter of the diurnal skin depth.

\section{Interpretation}

The different sensors reacted to the transits in distinct ways. The clearest signal is from the Phobos transit on Sol 501, which lasted $27 \mathrm{~s}$. In the following, we discuss the signals from this transit in the $135 \mathrm{~s}$ long window of Figure 1 and Table 1.

- Barometric pressure and atmospheric temperature: no reaction.

- Solar array current: Gaussian-like reduction for the duration of transit.

- Magnetometer: two components $\left(B_{\mathrm{x}}, B_{\mathrm{z}}\right)$ showed a Gaussian-like decrease very similar to the solar array current, while $B_{\mathrm{y}}$ showed no reaction. No delay in time between reaction of magnetometer relative to solar array current.

- Surface temperature: initial response as fast as for solar array current and magnetometer followed by a recovery phase of $\sim 1.5 \mathrm{~min}$. 

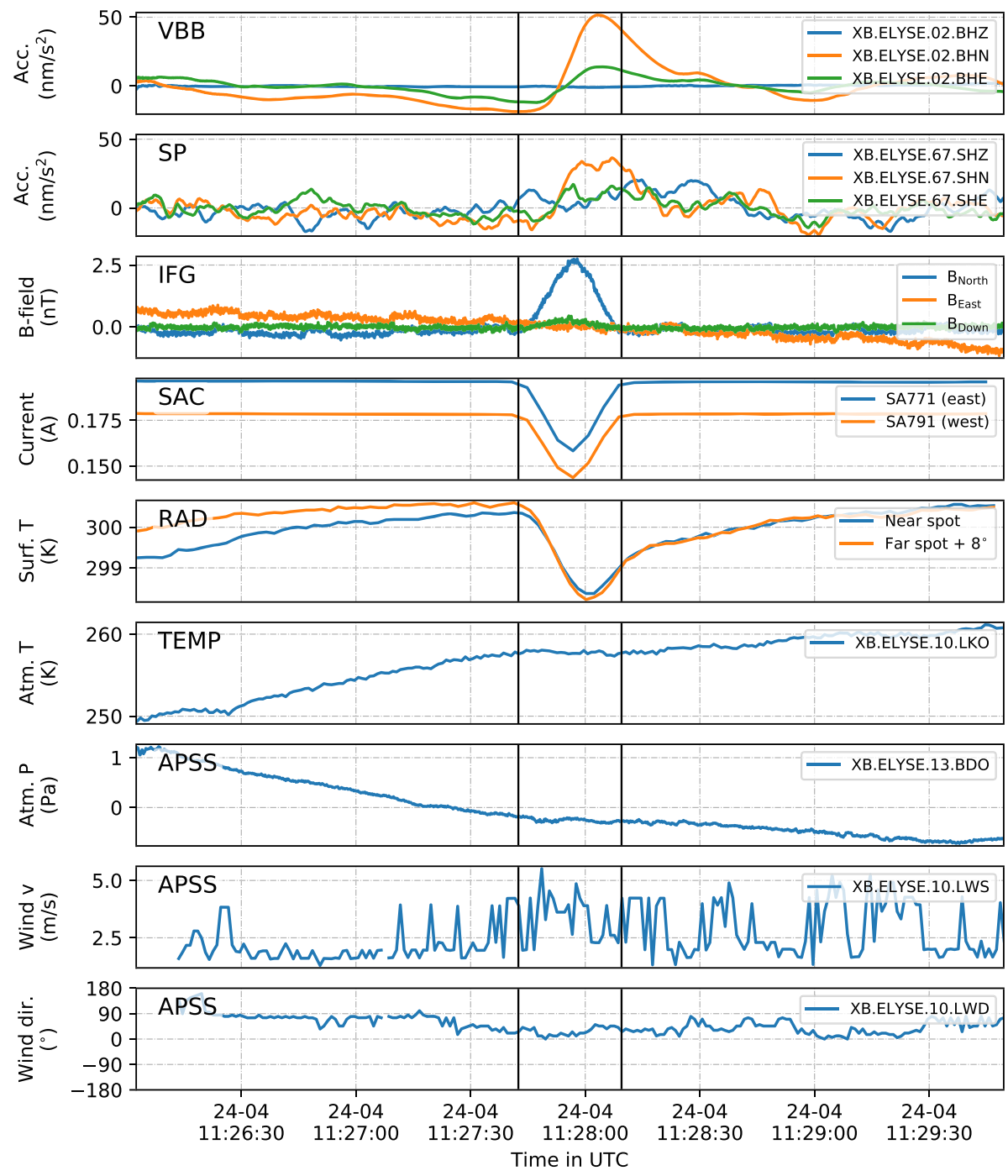

Figure 1. Data recorded during the Phobos transit at the InSight location on Sol 501 (24 April 2020). Channels are from top: band-pass filtered acceleration data (first-order Butterworth, 0.005-0.2 Hz) for the VBB and SP seismometers, demeaned magnetic data (IFG: InSight Fluxgate Magnometer), solar array currents (SAC), radiometer (RAD) surface temperatures of the near and far spot (see Mueller et al., 2020), as well as atmospheric temperature, atmospheric pressure, wind speed, and wind direction (pos. is clockwise from North) as part of the Auxiliary Payload Sensor Suite (APSS, Banfield et al., 2018). Black vertical lines: eclipse start and end times according to the JPL ephemeris mar097 (Jacobson \& Lainey, 2014). Note that atmospheric temperature and pressure as well as the wind show no significant changes during the eclipse. For the details of the channel naming, see Lognonné et al. (2019).

- Broadband (VBB) seismometer: clear reaction of U, V, and $\mathrm{W}$ components. After transformation into canonical $\mathrm{Z}, \mathrm{N}$, and $\mathrm{E}$ components and removal of the instrument response, we observe a purely horizontal acceleration (Table 1 and Figure S2) into NNE direction, which is delayed relative to the magnetometer, and solar array currents by $5 \mathrm{~s}$. A recovery phase of $\sim 1$ min follows.

- SP seismometer (Figure S2): reaction is compatible with VBB but with lower signal-to-noise ratio.

How can we understand these different reactions? The array current is the most straightforward to interpret: During the transit, the solar disk is partially covered by Phobos, and hence less radiation reaches the solar arrays. This signal is probably the most direct evidence for the transit considering that the zoom level of the InSight cameras does not allow for a picture of the Sun with high enough resolution (Maki et al., 2018). 
The magnetic field variations align perfectly with a scaled version of the array currents. The two wave forms are so similar that a delay of $1 \mathrm{~s}$ would be detectable. As pointed out above, this lack of delay is a strong indication for a cross-talk from the solar array currents leading to an electronically induced magnetic signal.

The radiometer provides a direct observation of the cooling of the Martian surface during the transit. The cooling amounts to $\Delta T=2.5 \mathrm{~K}$ for the events on Sols 97 and 501. The skin depth for such a short, 30-s negative heat pulse is only $0.5-1.0 \mathrm{~mm}$.

The response of SEIS to the Phobos transit consists of an apparent horizontal acceleration which is delayed relative to the current output of the solar arrays by $5 \mathrm{~s}$ (Table 1). We have considered several hypotheses to explain this reaction of SEIS to the transits that we list and discuss in the following:

(A) gravity and pressure signal from atmospheric cooling

(B) contraction of the tether

(C) magnetic sensitivity of VBB leaf springs

(D) thermal leak by convection through contact zone between the wind-thermal-shield (WTS) and soil with subsequent contraction of the seismometer legs

(E) thermal conduction through WTS and the seismometer insulation (RWEB)

(F) thermal effect on feedback electronics

(G) tidally triggered seismicity

(H) thermoelastic response of subsurface

(A) A change in atmospheric temperature across the entire air column above the lander would lead to a change in density and subsequently to a change in pressure. This hypothesis can be discarded because, on the one hand, the barometer did not show any response and, on the other hand, the reaction of the seismometer would show up primarily on the vertical seismometer component: the Newtonian upward acceleration exerted by the high density air masses above the InSight lander (Zürn \& Widmer-Schnidrig, 1995). (B) A contraction of the tether would lead to a force pulling at the load shunt assembly (LSA) toward the lander. SEIS would then tilt toward the lander, opposite to the observation. We thus reject this hypothesis. (C) The magnetic sensitivity of the VBB leaf springs is on the order of $0.5 \mathrm{nT} /\left(\mathrm{nms}^{-2}\right)$ (Lognonné et al., 2019). Even when assuming that the same $B$-field perturbations occurred at the locations of both the magnetometer and SEIS, which is unrealistic, if they are caused by the solar-array current, the magnetic field perturbations (Table 1) of $3 \mathrm{nT}$ would only create a VBB signal of $6 \mathrm{~nm} / \mathrm{s}^{2}$, which is an order of magnitude too small (Table 1). Furthermore, we would expect a perfect match of the wave forms (Forbriger, 2007), which is not what we observe. So there are multiple reasons to discard this hypothesis. (D) The time constants involved are too large: hours rather than seconds (Mimoun et al., 2017). Note also that each of the seismometer legs is thermally protected by its own bellows (Lognonné et al., 2019). (E) Same as for (D). (F) The feedback electronics are stored inside the lander in a heated compartment. The temperature change during the eclipse was below measurement uncertainty. (G) The observed signal does not have any similarity with observed marsquakes, especially no highly scattered coda (Giardini et al., 2020). Again, this hypothesis can be rejected. $(\mathrm{H})$ The hypothesis of a thermoelastic response of the ground and subsequent tilting of the seismometer derives from a fortuitous observation that was made at the Black Forest Observatory (BFO) in 1997 (Zürn \& Otto, 2000): When leaving the seismometer vault, the technician forgot to switch off the light and later on noticed that the noise level of the long-period data was elevated since the last visit to the vault. This triggered a long series of experiments with artificial heat sources (light bulbs and soldering irons) in the BFO seismometer vault that established that well-shielded horizontal long-period seismometers react almost instantaneously to heat input to the seismic pillar. Thermoelastic strains were the only plausible physical mechanism by which these experiments could be explained.

We interpret the observed apparent horizontal acceleration toward NNE as the seismometer response to a tilt down in the SSW direction. The tilt response of the seismometer to Phobos and Deimos transits is shown in Table 1. Only the three transits with the highest solar elevation generated a measurable response in the seismometer. Although the azimuth of the Sun varied between $126^{\circ}$ and $243^{\circ}$ and the direction toward the main shadow by $7^{\circ}$, the azimuth of the tilt only varied between $200^{\circ}$ and $211^{\circ}$.

A simple model to test the tilt hypothesis simulates shaded areas below the lander and the WTS that do not change temperature, while the surface everywhere else cools by a few degrees. This causes a thin layer to 
(a)
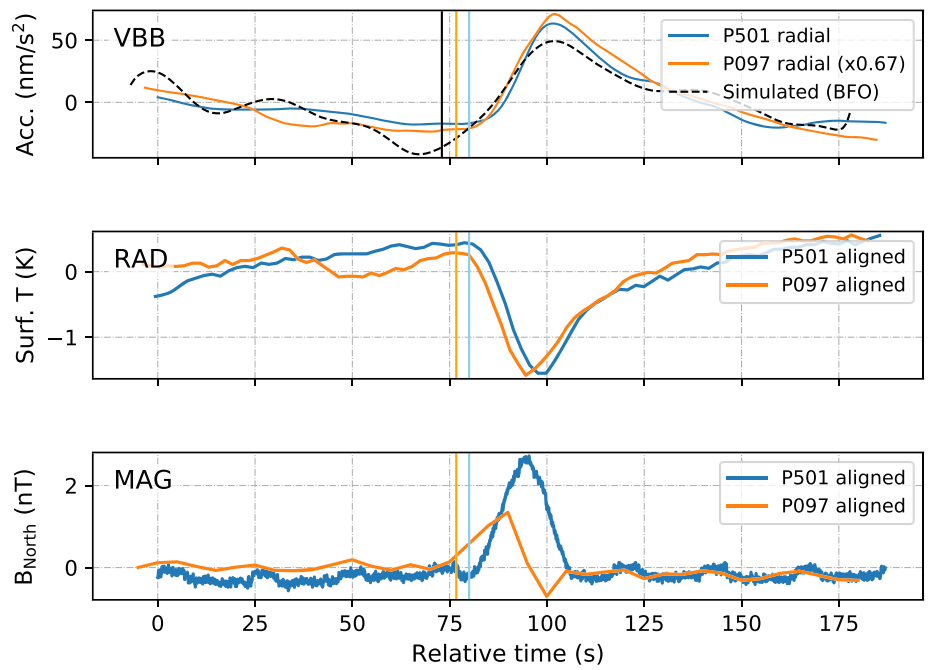

(b)
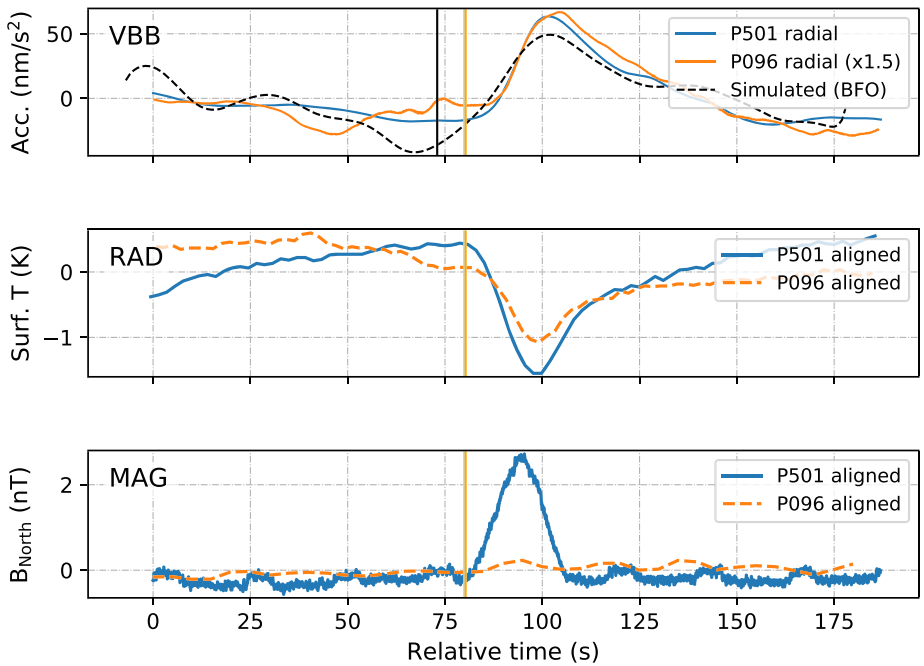

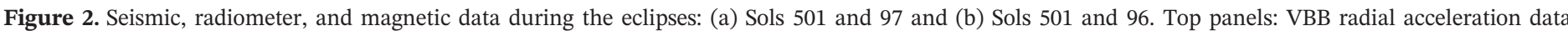

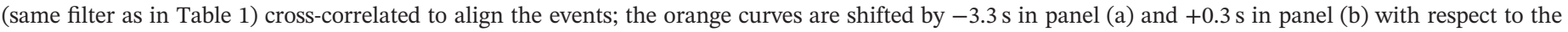

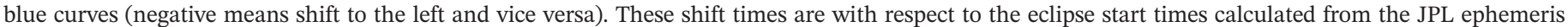

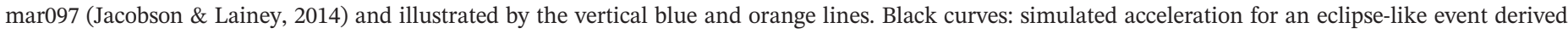
from our light bulb experiment in the seismometer vault at the Black Forest Observatory (BFO; see supporting information) with the black vertical line

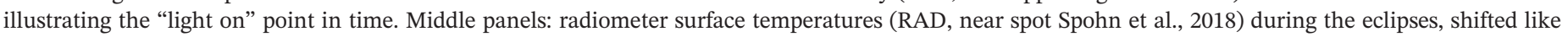

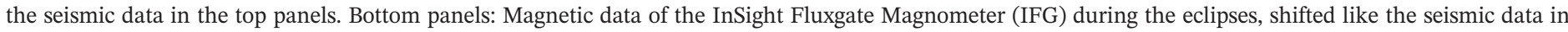

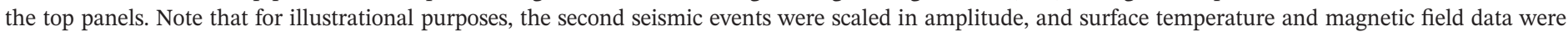
demeaned.

thermoelastically contract outside the shaded areas, where RAD is measuring the change in surface temperature (Figure 2). The penetration depth for this temperature perturbation is $0.5-1.0 \mathrm{~mm}$. This contracting surface layer is elastically coupled to the material below, causing thermoelastic stress, strain, tilt, and displacement fields in the top few meters, to which the seismometer responds.

\subsection{Analog Experiment at BFO}

Since the Phobos transit is a rapid event, it is not easy to find a terrestrial analog: On Earth, solar transits take about $3 \mathrm{hr}$, such that the response is well outside the pass-band of seismometers. Therefore, we chose to simulate the Phobos transit with an experiment in a well-controlled environment of a quiet seismic vault at the BFO near Schiltach, Germany. The question we try to answer is if shining a light on a well-shielded seismometer can lead to a response at time scales of only a few seconds. Thus we repeat the experiment of Zürn and Otto (2000) with improved timing accuracy for the switching of the light source. We observe the differential signal of two VBB seismometers, one shielded with a 1.2-m wide styrofoam cube, but in line of sight of the bulb, and the other one installed in a separate vault $100 \mathrm{~m}$ away in a (dark) post-hole. The difference of the signals of the two sensors is free of noise from marine microseism and semidiurnal tides. Separate power circuits were used for instruments and light, and the experiment was done hours after human operators had left the cavern. The details of the experiment are described in the supporting information. We find that the signal following the switching of the light bulb is broadly consistent with the Phobos transit response of SEIS on Mars: (1) No signal is recorded on the vertical component, consistent with tilt. (2) The signal on the horizontal channels has a delay to the input of $12 \mathrm{~s}$, compared to the $5 \mathrm{~s}$ on Mars. (3) A thermal conduction effect is observed only with a time constant of $3 \mathrm{hr}$, that is, much longer than the duration of the transit on Mars.

\subsection{Finite Element Modeling}

We use a finite element model (FEM) to better understand the amplitude elastic response to the thermally induced stresses at the surface due to the cooling in the regions that suddenly see a drop in solar irradiation. In the shadows of the WTS and the lander with its solar panels and at depth, the temperature remains constant. We assume an exponential temperature profile with skin depth of $2 \mathrm{~mm}$, a temperature change at the 

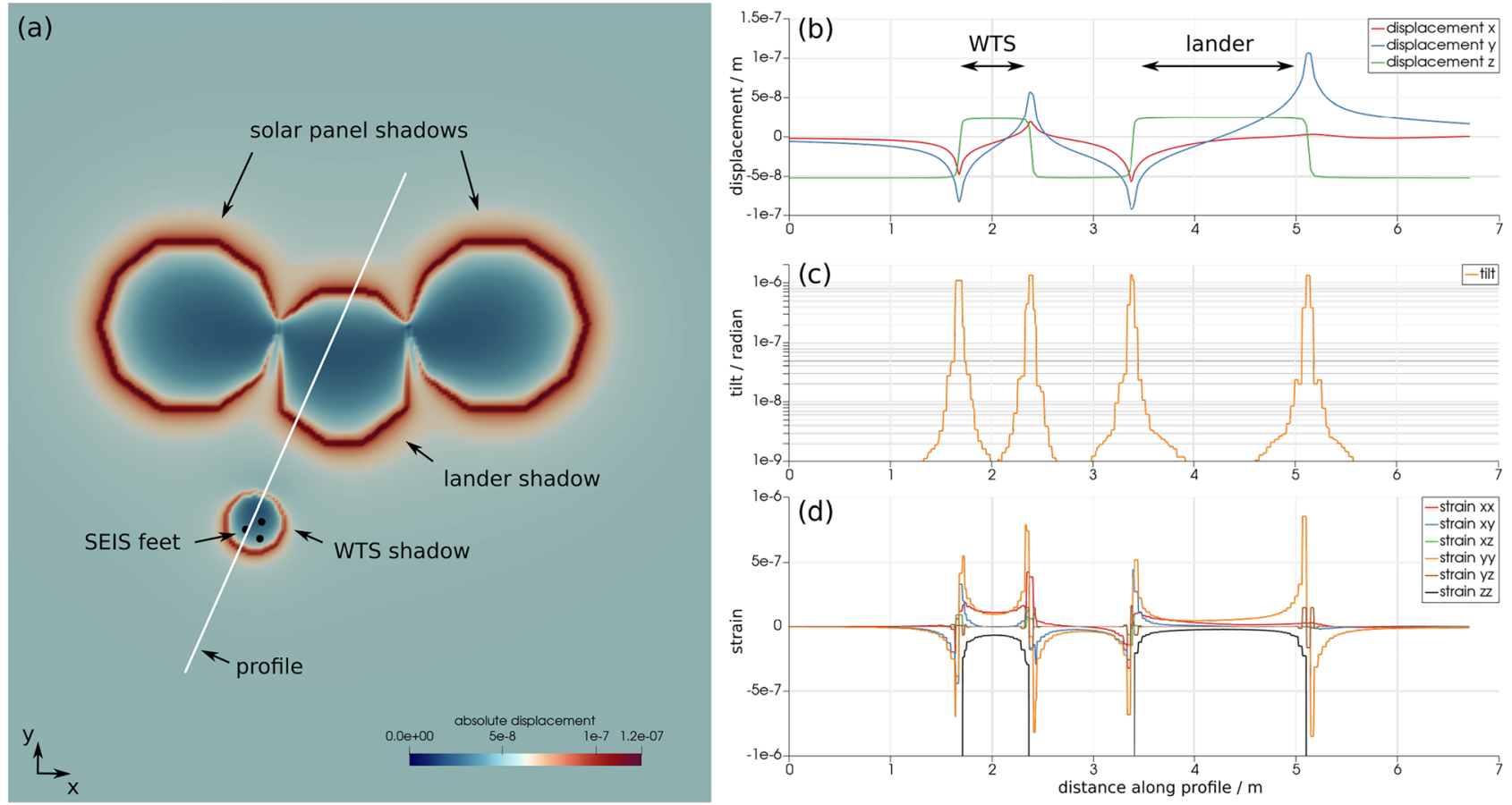

Figure 3. Finite element simulation of thermoelastic deformation in a medium with homogeneous elastic parameters for the shadows of P501. (a) Absolute value of the displacement, location of the SEIS feet, and indication of the profile used in panels (b)-(d). (b) Three-component displacements, (c) tilt, and (d) strains observed along the profile.

surface of $2 \mathrm{~K}$, a thermal expansion coefficient of $10^{-5} / \mathrm{K}$, and a Poisson ratio of 0.25 and use a domain of $(40 \mathrm{~m})^{3}$. The horizontal and vertical resolution in the region of interest are 35 and $0.8 \mathrm{~mm}$, respectively, and element sizes increase with distance (using the algorithm described in van Driel et al., 2020), available in the Salvus software suite). The top surface of the domain is stress free, the bottom boundary is fixed, and the four lateral boundaries allow motion only in parallel to the boundary. The isotropic thermal stresses are computed from the assumed thermal profile and then used as the right-hand side in the elastostatic equation, which is solved using the finite element method (Schaa et al., 2016).

The results in Figures $3 \mathrm{a}$ and $3 \mathrm{~b}$ demonstrate that the most relevant vertical displacement follows a surprisingly simple pattern with uplift in the shadow and suppression in the previously Sun-exposed areas and a steep transition between the two regimes within a few centimeters. While the horizontal displacements reach further out, we would not be able to observe them. The tilts associated with the vertical displacement pattern (Figure 3c) are concentrated at the shadow boundaries and quickly decay to very small values, including at the location of the SEIS feet. The relative vertical motion of the SEIS feet in this model is at least 1 order of magnitude smaller than the observation. On the other hand, the strains (Figure 3d) are nonzero below SEIS and in fact an order of magnitude larger in absolute value than the observed tilt (measured in radians). As a consequence, any heterogeneity or surface topography that causes even small coupling between strains and tilts (e.g., Harrison, 1976; van Driel et al., 2012) is more likely to cause the observed tilts than the prediction from a homogeneous half space model. These strain coupled tilts can take any direction, but as the effect is linear, the direction should be similar for similar shadowing.

\subsection{Timing of the Transit}

From the transits signature on the various instruments, it is possible to compare the timing of the Phobos transits with their expected timing from ephemerides models used for the orbit of Phobos. Relative to mar097 (Jacobson \& Lainey, 2014), the measured timing of maximal depth in the light curve, as determined from the solar array current, is $8 \mathrm{~s}$ early for the Sol 495 transit, $5 \mathrm{~s}$ late for the Sol 498 transit, $0.5 \mathrm{~s}$ late for the Sol 499 transit, and $0.5 \mathrm{~s}$ early for the Sol 501 transit, with fit uncertainties of at best $\pm 0.5 \mathrm{~s}$. As the errors over $1 \mathrm{~s}$ are associated with the low-Sun events in which insulation was dominated by diffuse light, it seems 
unlikely that they imply errors in the ephemerides. Rather, scattered light responds to the shadow in the dusty atmosphere early as Phobos sets in front of the rising Sun and late for the reverse. Therefore, the errors in the timing of the maximal depth in the light curves are likely to be due to some atmospheric effects such as dust scattering. Besides, all modeled light curve transits can be fitted with the observations without changing their duration with an accuracy better than $1 \mathrm{~s}$ (for a time sampling of one data point every $4 \mathrm{~s}$ ). This seems to indicate that the mar097 ephemerides are correct in their predictions. Since the accuracy of the Phobos ephemerides should be better than several hundred meters (Jacobson, 2010; Jacobson \& Lainey, 2014), this would translate into a timing error of less than 0.5 s given Phobos' orbital speed. Further work is needed to study the relation between the observed offsets and the observed depths together with the local time and the Sun's position to model these atmospheric effects and constrain the dust in the Martian atmosphere during the Phobos transits for a given set of Phobos ephemerides. Since the SEIS sensors are by far the ones with the highest sampling rate to ever have observed an transit on Mars (20 sps for the VBB seismometer and 100 sps for SP), we tried to use the signals of different transits as matched filters to estimate the time difference between two following transits (Figure 2). The timing difference between VBB and RAD or MAG is always larger than the difference between the latter two. This is to be expected, since the effect on VBB is delayed by the thermal conduction of the cooling in the near surface.

\section{Discussion}

Of all signals, the solar array currents are the easiest to explain, followed by the surface temperature, as detected by the radiometer. Given that the magnetic field signal matches the timing of the solar array currents and that both are absent for near-miss transits, we conclude that the IFG signals are most likely generated by changes in the solar array currents. They can therefore serve to measure the timing of transits, at times when SAC was only recorded every $30 \mathrm{~s}$.

The observed VBB accelerations are compatible with a tilt of SEIS. Furthermore, the onset of the VBB signal is delayed in time by $5 \mathrm{~s}$ relative the the solar array currents, the radiometer, or the magnetometer. Both of these observations match with what was observed in the BFO seismometer vault experiment: broadband seismometers that are very well isolated from heat conduction and from direct solar radiation can still respond within seconds to external irradiation, an observation that we can only explain with a thermoelastic response of the ground and associated tilting of the seismometer. What is responsible for the thermoelastic deformation? Is it the thermal contrast created by the shadow of the WTS or the lander and its solar arrays? What argues in favor of the lander shadow hypothesis is that it is larger than the WTS shadow and that the tilt azimuths point away from the lander. However, the FE modeling for a homogeneous half space predicts that the tilt generated by the lander shadow is too small to explain the observed tilt. A more likely scenario based on the predictions of the FE calculations is that the WTS shadow is responsible for the observed tilt. However, even under the WTS, the tilts predicted by the FE modeling are not what SEIS sensed, but instead, we propose that strain coupled tilts due to very local heterogeneities generated the observed tilts. This is because the strains under the WTS are more than an order of magnitude larger than the predicted tilts. This has implications for surface-installed seismometers. It seems necessary not only to isolate the seismometer itself but also to cover the ground at least a meter around it. Further research will need to focus on modeling detailed layered models of the subsurface, the likes of which are not available on Mars yet.

\section{Summary}

We report the observation of eight solar transits by the sensors on the InSight lander. We use these well-defined events to better understand the Martian environment and how it responds to rapid changes in solar irradiation. The solar array currents have responded to all transits. They are the most sensitive detectors for such events. A drop in ground temperature has been detected for the larger transits, while no change in air temperature or barometric pressure was detected. The magnetometer most likely responded to the drop in array currents.

The VBB signals are the most complex ones to explain: temperature change in the shadow of the WTS creating thermoelastic strains in the duricrust which couples into tilt. The results will help to further constrain the timing of Phobos transits but also highlight the importance of strain-tilt coupling when modeling seismic responses. 


\section{Data Availability Statement}

All access points to the seismological data archives can be found at https://doi.org/10.18715/SEIS.INSIGHT. XB_2016. The data from the experiment at the Black Forest Observatory are available through the German Regional Seismic Network (GRSN; https://doi.org/10.25928/MBX6-HR74) and the IRIS/IDA seismic network (https://doi.org/10.7914/SN/II). The InSight data is on PDS for the 2019 transits and will be released to PDS for the 2020 transits in September 2020.

\section{Acknowledgments}

We acknowledge NASA, CNES, partner agencies and institutions (UKSA, SSO, DLR, JPL, IPGP-CNRS, ETHZ, IC, and MPS-MPG), and the operators of JPL, SISMOC, MSDS, IRIS-DMC, and PDS for providing SEED SEIS data. French teams acknowledge support from CNES as well as Agence Nationale de la Recherche (ANR-14-CE36-0012-02 and ANR-19-CE31-0008-08). The Swiss contribution in implementation of the SEIS electronics was made possible through funding from the federal Swiss Space Office (SSO) and the contractual and technical support of the ESA-PRODEX office. The MPS-MPG SEIS team acknowledges funding for development of the SEIS leveling system by the DLR German Space Agency. Numerical simulations were supported by a grant from the Swiss National Supercomputing Centre (CSCS) under project ID s922. A. M. and C. L. J. acknowledge partial support from the Canadian Space Agency. M. L., F. N., and R. L. acknowledge partial support from the InSight PSP program under Grants $80 \mathrm{NSSC} 18 \mathrm{~K}$ $1621,80 \mathrm{NSSC} 18 \mathrm{~K} 1627$, and $80 \mathrm{NSSC} 18$ K1626. We thank Stefanie Donner and an anonymous reviewer for their assistance in evaluating this paper. This paper is InSight Contribution Number 177.

\section{References}

Banerdt, W. B., Smrekar, S. E., Banfield, D., Giardini, D., Golombek, M., Johnson, C. L., et al. (2020). Initial results from the InSight mission on Mars. Nature Geoscience, 13, 183-189. https://doi.org/10.1038/s41561-020-0544-y

Banfield, D., Rodriguez-Manfredi, J. A., Russell, C. T., Rowe, K. M., Leneman, D., Lai, H. R., et al. (2018). Insight Auxiliary Payload Sensor Suite (APSS). Space Science Reviews, 215(1), 4. https://doi.org/10.1007/s11214-018-0570-x

Banfield, D., Spiga, A., Newman, C., Forget, F., Lemmon, M., Lorenz, R., et al. (2020). The atmosphere of Mars as observed by InSight. Nature Geoscience, 3, 190-198. https://doi.org/10.1038/s41561-020-0534-0

Bell, J. F., Lemmon, M. T., Duxbury, T. C., Hubbard, M. Y. H., Wolff, M. J., Squyres, S. W., et al. (2005). Solar eclipses of Phobos and Deimos observed from the surface of Mars. Nature, 436(7047), 55-57. https://doi.org/10.1038/nature03437

Betts, B. H., Murray, B. C., \& Svitek, T. (1995). Thermal inertias in the upper millimeters of the Martian surface derived using Phobos' shadow. Journal of Geophysical Research, 100(E3), 5285-5296. https://doi.org/10.1029/95JE00226

Bills, B. G., Neumann, G. A., Smith, D. E., \& Zuber, M. T. (2005). Improved estimate of tidal dissipation within Mars from MOLA observations of the shadow of Phobos. Journal of Geophysical Research, 110, E07004. https://doi.org/10.1029/2004JE002376

Christou, A. A. (2002). Lander position determination on Mars using Phobos transits: Application to Beagle 2. Planetary and Space Science, 50(7), 781-788. https://doi.org/10.1016/S0032-0633(02)00016-8

Duxbury, T. C. (1978). Phobos transit of Mars as viewed by the Viking cameras. Science, 199(4334), 1201-1202. https://doi.org/10.1126/ science.199.4334.1201

Folkner, W. M., Dehant, V., Le Maistre, S., Yseboodt, M., Rivoldini, A., Van Hoolst, T., et al. (2018). The rotation and interior structure experiment on the InSight mission to Mars. Space Science Reviews, 214(5), 100. https://doi.org/10.1007/s11214-018-0530-5

Forbriger, T. (2007). Reducing magnetic field induced noise in broad-band seismic recordings. Geophysical Journal International, 169, 240-258.

Garcia, R. F., Kenda, B., Kawamura, T., Spiga, A., Murdoch, N., Lognonné, P. H., et al. (2020). Pressure effects on the SEIS InSight instrument, improvement of seismic records, and characterization of long period atmospheric waves from ground displacements. Journal of Geophysical Research: Planets, 125, e2019JE006278. https://doi.org/10.1029/2019JE006278

Giardini, D., Lognonné, P., Banerdt, W. B., Pike, W. T., Christensen, U., Ceylan, S., et al. (2020). The seismicity of Mars. Nature Geoscience, 13, 205-212. https://doi.org/10.1038/s41561-020-0539-8

Golombek, M., Warner, N. H., Grant, J. A., Hauber, E., Ansan, V., Weitz, C. M., et al. (2020). Geology of the InSight landing site on Mars. Nature Communications, 11(1), 1014. https://doi.org/10.1038/s41467-020-14679-1

Golombek, M., Williams, N., Warner, N. H., Parker, T., Williams, M. G., Daubar, I., et al. (2020). Location and setting of the InSight lander, instruments and landing site. Earth, Planets and Space, 7, e2020EA001248. https://doi.org/10.1029/2020EA001248

Grier, J. A., \& Rivkin, A. S. (2019). Chapter 2-Common characteristics of airless bodies. In J. A. Grier \& A. S. Rivkin (Eds.), Airless bodies of the inner solar system (pp. 9-26). Amsterdam, Netherlands: Elsevier. https://doi.org/10.1016/B978-0-12-809279-8.00002-0

Harrison, J. C. (1976). Cavity and topographic effects in tilt and strain measurement. Journal of Geophysical Research, 81(2), 319-328.

Huba, J. D., \& Drob, D. (2017). Sami3 prediction of the impact of the 21 August 2017 total solar eclipse on the ionosphere/plasmasphere system. Geophysical Research Letters, 44, 5928-5935. https://doi.org/10.1002/2017GL073549

Jacobson, R. A. (2010). The orbits and masses of the Martian satellites and the libration of Phobos. The Astronomical Journal, 139(2), 668-679. https://doi.org/10.1088/0004-6256/139/2/668

Jacobson, R. A., \& Lainey, V. (2014). Martian satellite orbits and ephemerides. Planetary and Space Science, 102, 35-44. https://doi.org/ 10.1016/j.pss.2013.06.003

Johnson, C. L., Mittelholz, A., Langlais, B., Russell, C. T., Ansan, V., Banfield, D., et al. (2020). Crustal and time-varying magnetic fields at the InSight landing site on Mars. Nature Geoscience, 13, 199-204. https://doi.org/10.1038/s41561-020-0537-x

Joy, S. P., Mafi, J. N., \& Slavney, S. (2019). Interior Exploration Using Seismic Investigations, Geodesy, and Heat Transport (InSight) Mission. InSight Fluxgate Magnetometer (IFG). PDS Archive Software Interface Specification. urn:nasa:pds:insight-ifg-mars:document: insight-ifg-sis.

Kenda, B., Drilleau, M., \& Lognonne, P. (2020). Subsurface structure at the InSight landing site from compliance measurements by seismic and meteorological experiments. Journal of Geophysical Research: Planets, 125, e2020JE006387. https://doi.org/10.1029/2020JE006387

Khan, A., Liebske, C., Rozel, A., Rivoldini, A., Nimmo, F., Connolly, J. A. D., et al. (2018). A geophysical perspective on the bulk composition of Mars. Journal of Geophysical Research: Planets, 123, 575-611. https://doi.org/10.1002/2017JE005371

Kim, J.-H., \& Chang, H.-Y. (2018). Statistical analysis of geomagnetic field variations during solar eclipses. Advances in Space Research, 61(8), 2040-2049. https://doi.org/10.1016/j.asr.2018.01.022

Ledger, E. (1879). The eclipses of the satellites of Mars. The Observatory, 3, 191-193.

Lognonné, P., Banerdt, W. B., Giardini, D., Pike, W. T., Christensen, U., Laudet, P., et al. (2019). SEIS: InSight's seismic experiment for internal structure of Mars. Space Science Reviews, 215(1), 12. https://doi.org/10.1007/s11214-018-0574-6

Lognonné, P., Banerdt, W. B., Pike, W. T., Giardini, D., Christensen, U., Garcia, R. F., et al. (2020). Constraints on the shallow elastic and anelastic structure of Mars from InSight seismic data. Nature Geoscience, 13, 213-220. https://doi.org/10.1038/s41561-020-0536-y

Lorenz, R. D., Lemmon, M. T., Maki, J., Banfield, D., Spiga, A., Charalambous, C., et al. (2020). Scientific observations with the InSight solar arrays: Dust, clouds and eclipses on Mars. Earth and Space Science, 7, e2019EA000992. https://doi.org/10.1029/2019EA000992

Lorenz, R. D., Lemmon, M. T., \& Mueller, N. (2020). A transit lightcurve of Deimos, observed with the InSight solar arrays. Research Notes of the $A A S, 4(4), 57$. https://doi.org/10.3847/2515-5172/ab8d21

Maki, J. N., Golombek, M., Deen, R., Abarca, H., Sorice, C., Goodsall, T., et al. (2018). The color cameras on the InSight lander. Space Science Reviews, 214(6), 105. https://doi.org/10.1007/s11214-018-0536-z 
Mimoun, D., Murdoch, N., Lognonné, P., Hurst, K., Pike, W. T., Hurley, J., et al. (2017). The noise model of the SEIS seismometer of the InSight Mission to Mars. Space Science Reviews, 211(1-4), 383-428. https://doi.org/10.1007/s11214-017-0409-x

Mittelholz, A., Johnson, C. L., Thorne, S. N., Joy, S., Barrett, E., Fillingim, M., et al. (2020). The origin of observed magnetic variability for a sol on Mars from InSight. Journal of Geophysical Research: Planets, 125, e2020JE006505. https://doi.org/10.1029/2020JE006505

Mueller, N. T., Knollenberg, J., Grott, M., Kopp, E., Walter, I., Krause, C., et al. (2020). Calibration of the HP3 radiometer on InSight. Earth and Space Science, 7, e2020EA001086. https://doi.org/10.1029/2020EA001086

Nimmo, F., \& Faul, U. H. (2013). Dissipation at tidal and seismic frequencies in a melt-free, anhydrous Mars. Journal of Geophysical Research: Planets, 118, 2558-2569. https://doi.org/10.1002/2013JE004499

Reinisch, B. W., Dandenault, P. B., Galkin, I. A., Hamel, R., \& Richards, P. G. (2018). Investigation of the electron density variation during the 21 August 2017 solar eclipse. Geophysical Research Letters, 45, 1253-1261. https://doi.org/10.1002/2017GL076572

Schaa, R., Gross, L., \& Du Plessis, J. (2016). PDE-based geophysical modelling using finite elements: Examples from 3D resistivity and 2D magnetotellurics. Journal of Geophysics and Engineering, 13(2), S59-S73. https://doi.org/10.1088/1742-2132/13/2/S59

Scholz, J.-R., Barruol, G., Fontaine, F. R., Sigloch, K., Crawford, W., \& Deen, M. (2017). Orienting ocean-bottom seismometers from P-wave and Rayleigh wave polarisations. Geophysical Journal International, 208(3), 1277-1289. https://doi.org/10.1093/gji/ggw426

Schweitzer, J., Pirli, M., Roth, M., \& Kvaerna, T. (2014). TROLL: A new, very broadband seismic station in Antarctica. Seismological Research Letters, 85(4), 852-862. https://doi.org/10.1785/0220130223

Spiga, A., Banfield, D., Teanby, N. A., Forget, F., Lucas, A., Kenda, B., et al. (2018). Atmospheric science with insight. Space Science Reviews, 214(7), 109. https://doi.org/10.1007/s11214-018-0543-0

Spiga, A., Murdoch, N., Lorenz, R., Forget, F., Newman, C., Rodriguez, S., et al. (2020). A study of daytime convective vortices and turbulence in the martian Planetary Boundary Layer based on half-a-year of InSight atmospheric measurements and Large-Eddy Simulations. arXiv preprint arXiv:2005.01134. http://arxiv.org/abs/2005.01134

Spohn, T., Grott, M., Smrekar, S. E., Knollenberg, J., Hudson, T. L., Krause, C., et al. (2018). The heat flow and physical properties package (HP3) for the InSight mission. Space Science Reviews, 214(5), 96. https://doi.org/10.1007/s11214-018-0530-5

Usui, T., Kuramoto, K., \& Kawakatsu, Y. (2018). Martian moons exploration (MMX): Japanese Phobos sample return mission. In 42nd cospar scientific assembly, 42.

van Driel, M., Boehm, C., Krischer, L., \& Afanasiev, M. (2020). Accelerating numerical wave propagation using wavefield adapted meshes. Part I: forward and adjoint modelling. Geophysical Journal International, 221(3), 1580-1590. https://doi.org/10.1093/gji/ggaa058

van Driel, M., Wassermann, J., Nader, M.-F., Schuberth, B. S. A., \& Igel, H. (2012). Strain rotation coupling and its implications on the measurement of rotational ground motions. Journal of Seismology, 16, 657-668. https://doi.org/10.1007/s10950-012-9296-5

Zürn, W., \& Widmer-Schnidrig, R. (1995). On noise reduction in vertical seismic records below $2 \mathrm{mHz}$ using local barometric pressure. Geophysical Research Letters, 22(24), 3537-3540. https://doi.org/10.1029/95GL03369

Zürn, W., \& Otto, H. (2000). Lights or heat in the seismic vault, Black Forest Observatory (BFO). Internal Technical Report, 3. 